\title{
Clusters of Toxoplasmosis in Gyodong-Myeon and Samsan-Myeon, Ganghwa-Gun, Korea
}

\author{
Woojin Kim¹ ${ }^{1}$, Yoon Kyung Chang², Tong-Soo Kim³, Sung-Jong Hong ${ }^{4}$, Hye-Jin Ahn², Ho-Woo Nam², \\ Dongjae $\mathrm{Kim}^{1, *}$ (iD \\ 'Department of Biomedicine Health Science, College of Medicine, Catholic University of Korea, Seoul 06591, Korea; '2Department of Parasitology, \\ College of Medicine, Catholic University of Korea, Seoul 06591, Korea; ${ }^{3}$ Department of Parasitology and Tropical Medicine and Inha Research \\ Institute for Medical Sciences, Inha University School of Medicine, Incheon 22333, Korea; ' $D$ Department of Medical Environmental Biology, \\ Chung-Ang University College of Medicine, Seoul 06974, Korea
}

\begin{abstract}
The purpose of this study is to find out the clusters with high toxoplasmosis risk to discuss the geographical pattern in 2 islands of Gyodong-myeon and Samsan-myeon in Ganghwa-gun, Korea. Seroepidemiological data of toxoplasmosis surveyed using rapid diagnostic tests for the residents in 2 islands from 2010 to 2013 were analyzed to detect clusters of the infection. The cluster was investigated using the SatScan program which is based on Kulldorff's scan statistic. The clusters were found with $P$-values in each region analyzed in the program, and the risk and patient incidence of specific areas can be examined by the values such as relative risk and log likelyhood ratio. Jiseok-ri was found to be a cluster in Gyodong-myeon and Ha-ri was the cluster in Samsan-myeon. This findings can be used to monitor and prevent toxoplasmosis infections occurring in vulnerable areas.
\end{abstract}

Key words: Toxoplasma gondii, RDT, resident, cluster, Ganghwa-gun

\section{INTRODUCTION}

Toxoplasma gondii, an apicomplexan zoonotic protozoan, is one of the most widespread parasites worldwide. Warm blooded animals are its hosts, especially felids. Toxoplasmosis is acquired by the infection with $T$. gondii through undercooked meat, contaminated water, or mother to her fetus. Congenital toxoplasmosis may cause critical damages to the fetus [1-5]. For adult humans, most of their symptoms are light or asymptomatic. However, the infections can be exacerbated into chronic conditions and can cause a serious diseases in immune compromised patients [6-9]. Rapid diagnostic test (RDT) is a tool for diagnosis of toxoplasmosis. Seroprevalence of toxoplasmosis in certain areas is surging recently [10-13]. Previous studies have shown that the overall proportion, gender and age distribution of Gyodong-myeon and Samsan-myeon $[11,12]$. In addition to this, if clusters with high risk are specified by comparing them to each region, efficient monitor-

- Received 20 September 2020, revised 28 September 2020, accepted 29 September 2020.

*Corresponding author (djkim@catholic.ac.kr) (C) 2020, Korean Society for Parasitology and Tropical Medicine This is an Open Access article distributed under the terms of the Creative Commons Attribution Non-Commercial License (https://creativecommons.org/licenses/by-nc/4.0) which permits unrestricted non-commercial use, distribution, and reproduction in any medium, provided the original work is properly cited. ing and health policies can be established accordingly.

Spatial scan statistic and Kulldorffs SaTScan Software were used to detect the clusters. This study analyzed clusters using RDT for the detection of anti-T. gondii antibodies with the sera of residents in Gyodong-myeon, Samsam-myeon both in Ganghwa-gun, Incheon, Korea for 4 years from 2010 to 2013.

\section{MATERIALS AND METHODS}

\section{Ethics statement}

This study was performed under the regulation of the IRB Committee of The Catholic University of Korea (No. MIRB20200710-003). This research adhered to the tenets of the Declaration of Helsinki.

\section{Rapid diagnostic test (RDT)}

RDT data with address of subjects from Gyodong-myeon and Samsan-myeon was obtained from preceding studies, and the number of patients in each region is summarized in the Table 1 [11,12]. IgG/IgM RDT mounted with recombinant fragment of major surface antigen (SAG1A), GST-linker-SAG1A, were applied to the sera mentioned above [14]. Briefly, $10 \mu \mathrm{l}$ of serum was applied to the RDT sample hole and eluted with RDT buffer, i.e., $0.1 \%$ casein and $1 \%$ Tween 20 in $0.1 \mathrm{M}$ 
Tris-HCl buffer ( $\mathrm{pH} \mathrm{8.0)}$ a few seconds later. Reacting bands were read by 3 investigators after 15-20 $\mathrm{min}$, and its density was determined arbitrarily as $0,+,++$, and +++ . Definite reactivity was determined as ++ (mid), while weaker and stronger reactivity compared to it was determined as + (weak) and +++ (strong). The final results were agreed upon by the 3 investigators.

\section{Spatial scan statistic}

Spatial scan statistics continually change the size and location of predefined shaped windows and calculate the relative risk of risk within and outside the window for the region of interest, identifying clusters with relatively high risk compared to other regions [15]. Satscan increases the size gradually around each local unit, making circular windows that overlap each other hierarchical. Calculate the likelihood ratio for all windows and obtain the maximum likelihood ratio and use it as a test statistic. The Monte Carlo test is performed under the

Table 1. The number of sera, IgM-RDT positive number, and lgGRDT positive number of each regions in Gyodong-myeon and Samsan-myeon from 2010 to 2013

\begin{tabular}{lccrr}
\hline & Year & No. of sera & IgM-RDT (\%) & IgG-RDT (\%) \\
\hline Gyodong-myeon & 2010 & 921 & $3(0.3)$ & $191(20.7)$ \\
& 2011 & 999 & $1(0.1)$ & $243(24.3)$ \\
& 2012 & 929 & $7(0.8)$ & $251(27.0)$ \\
& 2013 & 848 & $13(1.5)$ & $226(26.7)$ \\
Samsan-myeon & 2010 & 313 & $1(0.3)$ & $84(26.8)$ \\
& 2011 & 342 & $1(0.3)$ & $107(31.3)$ \\
& 2012 & 379 & $29(7.7)$ & $137(36.1)$ \\
& 2013 & 361 & $5(1.4)$ & $183(50.7)$ \\
\hline
\end{tabular}

null hypothesis that the cluster does not exist $(\mathrm{RR}=1$, the risk is equal in all areas). First, we calculate the test statistic from the actual data to create a random set of data under the null hypothesis. The test statistics are calculated from each of these random repetitive datasets. Then, if it ranks in the upper alpha $\%$ by aligning the preceding test statistics, the null hypothesis is rejected under a significant level of alpha $\%$ and the $P$-value is calculated [16-19].

Shown in Table 1, a valuable cluster analysis of IgM-RDT cannot be obtained as the incidence of positive patients in IgM-RDT is significantly low, thus the analysis was only performed on IgG-RDT. Since the results of IgG-RDT are discrete at $0,+,++$, and +++ , and the proportion of positive patients is near or above $20 \%$, divided the results of IgG-RDT into positive and negative, and Bernoulli model used at $5 \%$ of significance level. The base point of each Ri shall be the latitude and longitude coordinates of the village hall (or community center) if there is no Ri office. If there are several halls, the coordinates of the midpoint were used. The window size is set to 25\%, 15\% per annum for the data in Gyodong-myeon and Samsan-myeon. In most cases, the upper cluster region and the relative risk were the same when the maximum allowed window was $15 \%$ and $25 \%$, while the $P$-value was lower when it was $15 \%$. Therefore, only clusters with a maximum allowed window size of $15 \%$ were used for the result analysis. In Table 2 and Table 3, relative risk indicates how much the risk of the cluster and surrounding areas varies. Log likelihood ratio is an indicator of how many times positive patients will occur in the cluster than a non-cluster. The greater the $P$-value, the less sig-

Table 2. Clusters detected from Gyodong-myeon by year and 4-year average

\begin{tabular}{|c|c|c|c|c|c|c|}
\hline Year & Cluster no. & Location & Case & Relative risk & Log likelihood ratio & $P$-value \\
\hline \multirow[t]{3}{*}{ Average } & 1 & Jiseok & 25 & 1.734 & 4.15 & 0.032 \\
\hline & 2 & Muhak & 15 & 1.175 & 0.236 & 0.995 \\
\hline & 3 & Yanggap & 20 & 1.15 & 0.23 & 0.997 \\
\hline \multirow[t]{2}{*}{2010} & 1 & Jiseok & 25 & 1.951 & 5.533 & 0.01 \\
\hline & 2 & Bongso & 20 & 1.247 & 0.527 & 0.929 \\
\hline \multirow[t]{3}{*}{2011} & 1 & Muhak & 41 & 1.473 & 3.206 & 0.103 \\
\hline & 2 & Bongso & 21 & 1.43 & 1.526 & 0.489 \\
\hline & 3 & Yanggap & 23 & 1.288 & 0.844 & 0.809 \\
\hline \multirow[t]{3}{*}{2012} & 1 & Jiseok & 29 & 1.654 & 4.174 & 0.035 \\
\hline & 2 & Sangyong & 22 & 1.256 & 0.69 & 0.877 \\
\hline & & Bongso & & & & \\
\hline \multirow[t]{2}{*}{2013} & 1 & Jiseok & 24 & 1.913 & 5.733 & 0.006 \\
\hline & 2 & Seohan & 20 & 1.259 & 0.638 & 0.919 \\
\hline
\end{tabular}


nificant the cluster is. Conversely, the smaller the $P$-value, the more certain the area is a cluster. The $\mathrm{R}$ program was used to visualize clusters on the map.

\section{RESULTS}

Using Satscan, the average and annual clusters of each region of Gyodong-myeon and Samsan-myeon were analyzed and marked on the map according to the relative risk of each cluster. A cluster with a $P$-value less than 0.05 is significant, but

Table 3. Clusters detected from Samsan-myeon by year and 4-year average

\begin{tabular}{lclrccc}
\hline Year & $\begin{array}{c}\text { Cluster } \\
\text { no. }\end{array}$ & Location & Case & $\begin{array}{c}\text { Relative } \\
\text { risk }\end{array}$ & $\begin{array}{c}\text { Log } \\
\text { likelihood } \\
\text { ratio }\end{array}$ & P-value \\
\hline Average & 1 & Ha & 24 & 1.711 & 3.945 & 0.002 \\
& 2 & Seogeom & 15 & 1.17 & 0.235 & 0.609 \\
& 3 & Sang & 8 & 1.178 & 0.146 & 0.748 \\
2010 & 1 & Ha & 42 & 1.886 & 3.833 & 0.015 \\
& 2 & Sang & 21 & 1.07 & 0.017 & 0.856 \\
2011 & 1 & Seogeom & 15 & 1.165 & 0.214 & 0.685 \\
& 2 & Sang & 8 & 1.071 & 0.025 & 0.89 \\
2012 & 1 & Ha & 29 & 1.993 & 8.469 & $<0.001$ \\
& 2 & Seogeom & 23 & 1.28 & 0.998 & 0.322 \\
& 3 & Sang & 3 & 1.19 & 0.068 & 0.885 \\
2013 & 1 & Ha & 20 & 1.433 & 1.63 & 0.102 \\
& 2 & Sang & 14 & 1.379 & 0.961 & 0.341 \\
& 3 & Seogeom & 14 & 1.242 & 0.441 & 0.584 \\
\hline
\end{tabular}

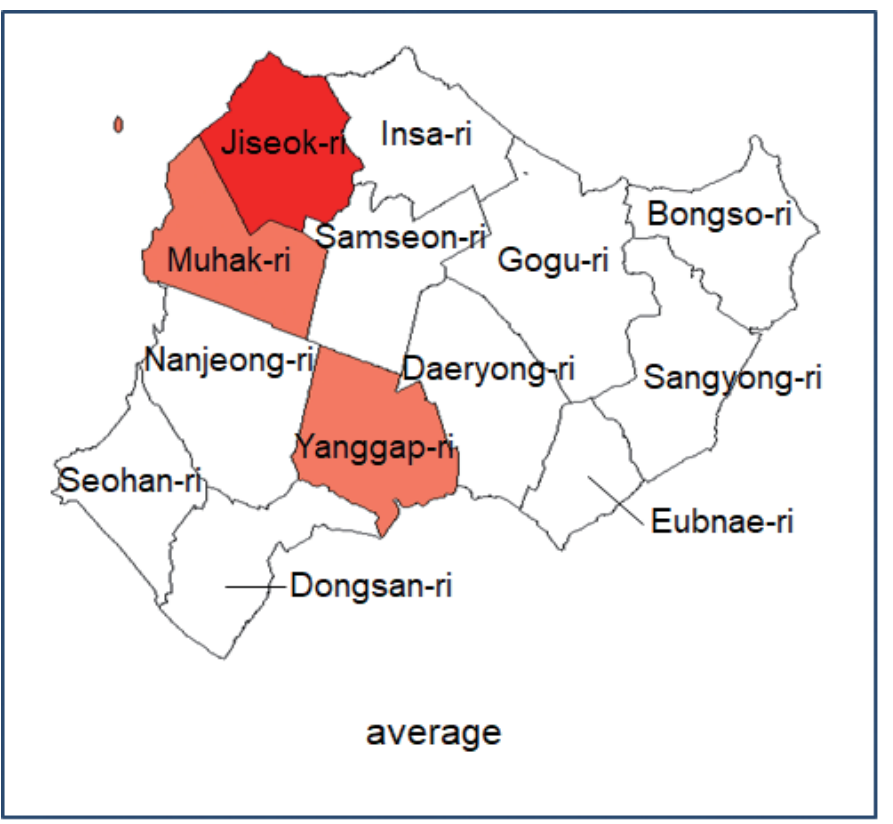

all clusters are plotted for visual comparison. If the cluster contains multiple regions, all are marked.

\section{Gyodong-myeon}

In the 4-year average of Gyodong-myeon, Jiseok-ri is the most likely cluster and is significant at a significant level of $5 \%$, with Log likelihood ratio $=4.150$, Relative risk $=1.734$, and $P$-value $=0.032$ $(<0.05)$, indicating the risk in Jiseok-ri was 1.734 times higher than other area (Table 2). Muhak-ri is the secondary and Yanggapri is the third cluster (both of $P$-value $>0.05$ ). In 2010, Jiseok-ri was also the most likely cluster with Log likelihood ratio $=5.533$, Relative risk $=1.951, P$-value $=0.01(<0.05)$, indicating the risk in Jiseok-ri was 1.951 times higher than other area. Bongso-ri and Gogu-ri are second and third, respectively. In 2011, Muhak-ri had Log likelihood ratio $=3.206$, Relative risk $=1.473, P$-value $=0.103$, which makes it hard to say that the first cluster because $P$-value is greater than 0.05. Bongso-ri is the second cluster and Yanggap-ri is the third. In 2012, Log likelihood ratio $=4.174$, Relative risk= 1.654, $P$-value $=0.035(<0.05)$ in Jiseok-ri, the first cluster. The second is Sangyong-ri, the third is Yanggap-ri and both $P$-value exceeded 0.05. In 2013, Log likelihood ratio $=5.733$, Relative risk $=$ $1.913, P$-value $=0.006(<0.05)$ Jiseok-ri is the most likely cluster and Seohan-ri is the second. Except for 2011, which falls short of the $P$-value standard, Jiseok-ri consistently appears as a cluster in Gyodong-myeon (Fig. 1).
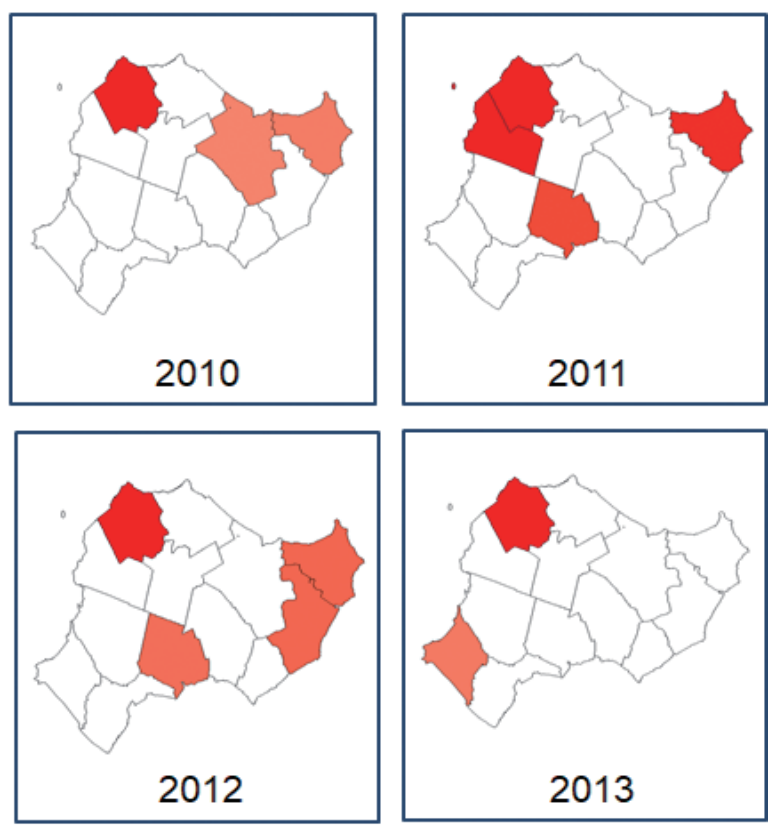

Fig. 1. Toxoplasmosis clusters in Gyodong-myeon by year. The higher the relative risk, the red the darker. 

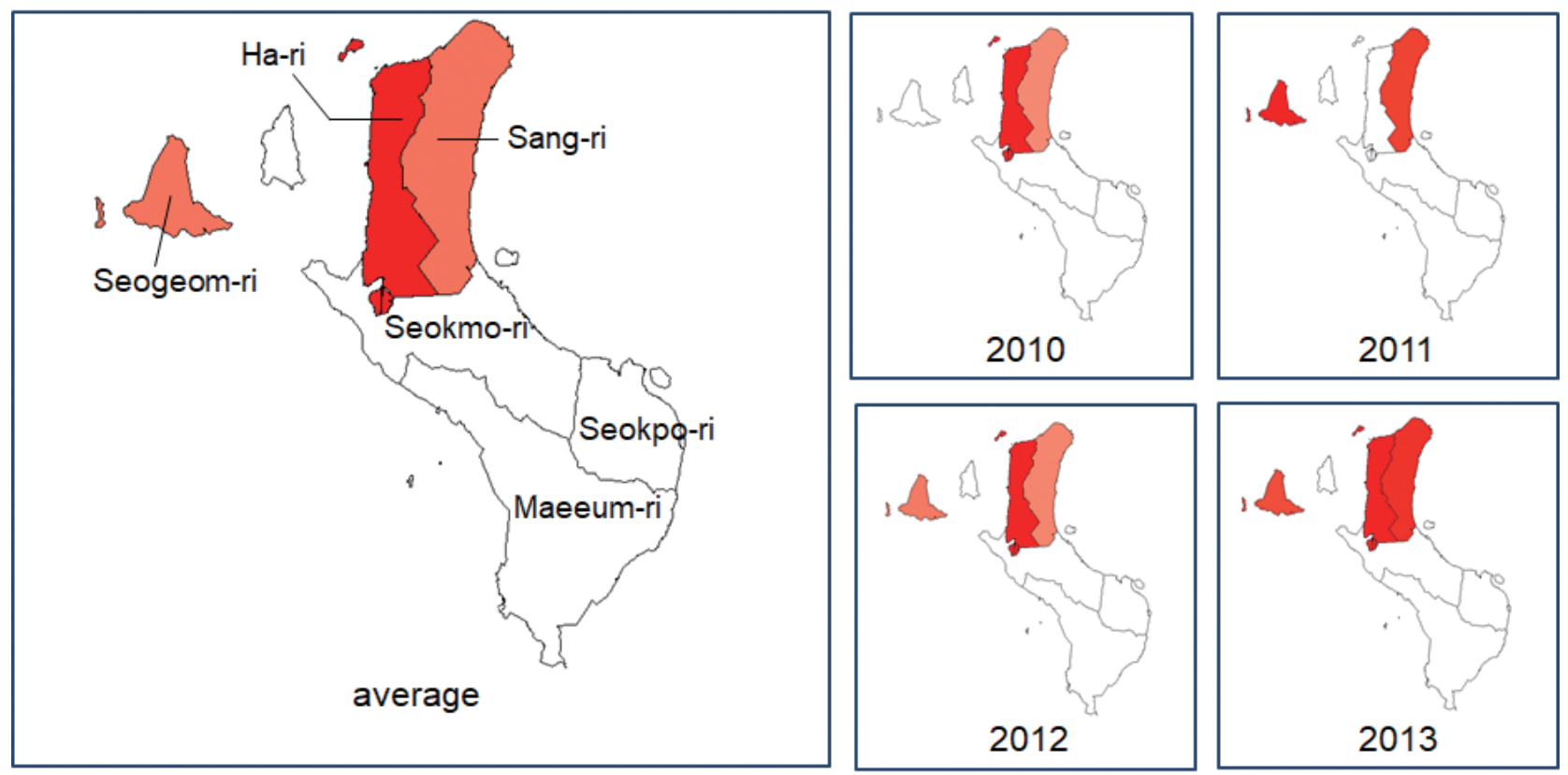

Fig. 2. Toxoplasmosis clusters in Samsan-myeon by year. The higher the relative risk, the red the darker.

\section{Samsan-myeon}

In the 4-year average of Samsan-myeon, Ha-ri is significant as the most likely cluster with $\mathrm{LLR}=3.955, \mathrm{RR}=1.71$ and $P$ value $=0.002(<0.05)$, indicating the risk in Ha-ri was 1.71 times higher than other area (Table 3). The second cluster is Seogeom-ri and the third is Sang-ri. In 2010, the most likely cluster is Ha-ri for $L L R=3.833$, Relative risk $=1.886$, and $P$-value $=0.015(<0.05)$, indicating the risk in Ha-ri was 1.886 times higher than other area, and Sang-ri is the second cluster. In 2011, the result is not taken into account because the $P$-value of the primary cluster, Seogeom-ri, is 0.685 , well above 0.05. Sang-ri is the second in 2011. In 2012, Ha-ri is the most likely cluster with $L L R=8.469, \mathrm{RR}=1.993$, and $P$-value is less than 0.001 , indicating the risk in Ha-ri in 2012 was 1.993 times higher than other area. Seogeom-ri is the second, Sang-ri is the third. In 2013, Ha-ri is primary cluster but $P$-value fell short of the standard at 0.102 . Sang-ri and Seogeom-ri are second and third, respectively. In the results of the Satscan analysis in Samsan-myeon, the $P$-value of all the second and third clusters is greater than 0.05 , that is, those are not statistically insignificant. Except for 2011 and 2013, the cluster in Samsanmyeon is Ha-ri (Fig. 2).

\section{DISCUSSION}

This study provided clusters with a high risk of toxoplasmo- sis infection in Gyodong-myeon and Samsan-myeon, Ganghwa-gun, Incheon, Korea through spatial scan analysis from 2010 to 2013. At the maximum allowed window size of $15 \%$, it was generally shown as Jiseok-ri is the most likely cluster in Gyodong-myeon for 4 years. In Samsan-myeon, the most likely cluster is Ha-ri.

Gyodong-myeon is the northernmost island of the Military Demarcation Line. Most of the islands are surrounded by iron fences, most residents are engaged in agriculture rather than fishing. There is a wharf on the east side of the island, and recently it is connected to Ganghwa-do island by yeonpyeong bridge. Gyodong-island is a region where toxoplastic lymphadenitis had a group outbreak in residents who ingested pork raised at home in 1997 [7]. Micro cluster in the island is located in the village in the northwest, and toxoplasmic enironment by cats is maintained until now. It is estimated that the better conditions are presented for the spread of infectious sources due to the recent introduction of organic farming methods in agricultural areas.

Samsan-myeon is the administrative district of Seokmododo, where the cluster is identified with a similar pattern to Gyodong-myeon above. Micro-clusters are formed in the northern agricultural area of the island rather than in the area near the wharf in the southwest and recently connected to the main island of Gangwha-do by the bridge.

Therefore, cluster analysis in relatively narrow islands in this 
study can estimate whether the source of $T$. gondii is oocyst mediated by cats, or tissue-cyst mediated by food and mainly pork, and can exclude T. gondii infection environments from farming structures. The findings can be used to monitor and prevent toxoplasmosis infections occurring in vulnerable areas.

\section{CONFLICT OF INTEREST}

The authors declare no conflict of interest related to this study.

\section{REFERENCES}

1. Black MW, Boothroyd JC. Lytic cycle of Toxoplasma gondii. Microbiol Mol Biol Rev 2000; 64: 607-623.

2. Furtado JM, Smith JR, Belfort R Jr, Gattey D, Winthrop KL. Toxoplasmosis: a global threat. J Glob Infect Dis 2011; 3: 281-284.

3. Tenter AM, Heckeroth AR, Weiss LM. Toxoplasma gondii: from animals to humans. Int J Parasitol 2000; 30: 1217-1258.

4. Montoya JG, Remington JS. Management of Toxoplasma gondii infection during pregnancy. Clin Infect Dis 2008; 47: 554-566.

5. Song KJ, Shin JC, Shin HJ, Nam HW. Seroprevalence of toxoplasmosis in Korean pregnant women. Korean J Parasitol 2005; 43: 69-71.

6. Weiss LM, Dubey JP. Toxoplasmosis: a history of clinical observations. Int J Parasitol 2009; 39: 895-901.

7. Choi WY, Nam HW, Kwak NH, Huh W, Kim YR, Kang MW, Cho SY, Dubey JP. Foodborne outbreaks of human toxoplasmosis. J Infect Dis 1997; 175: 1280-1282.

8. Kim MH, Choi YK, Park YK, Nam HW. A toxoplasmic uveitis case of a 60-year-old male in Korea. Korean J Parasitol 2000; 38: 29-31.

9. Park YH, Han JH, Nam HW. Clinical features of ocular toxoplasmosis in Korean patients. Korean J Parasitol 2011; 49: 167-171.
10. Ahn HJ, Cho PY, Ahn SK, Kim TS, Chong CK, Hong SJ, Cha SH, Nam HW. Seroprevalence of toxoplasmosis in the residents of Cheorwon-gun, Gangwon-do, Korea. Korean J Parasitol 2012; 50: 225-227.

11. Kim YH, Lee J, Ahn S, Kim TS. High seroprevalence of toxoplasmosis detected by RDT among the residents of Seokmo-do (Island) in Ganghwa-Gun, Incheon City, Korea. Korean J Parasitol. 2017; 55: 9-13.

12. Kim YH, Lee J, Ahn S, Kim TS. Seroprevalence of toxoplasmosis with ELISA and Rapid Diagnostic Test among residents in Gyodong-do, Inchon city, Korea: A Four-Year Follow-up. Korean J Parasitol. 2017; 55: 247-254.

13. Hong SJ, Chong CK, Lee K, Kim TS, Hong YP, Ahn HJ, Kim HY, Ko AR, Kim YJ, Nam HW. Maintained seroprevalence of toxoplasmosis among the residents of Jeju island, Korea. Korean J Parasitol 2011; 49: 309-311.

14. Song KJ, Yang Z, Chong CK, Kim JS, Lee KC, Kim TS, Nam HW. A rapid diagnostic test for toxoplasmosis using recombinant antigenic N-terminal half of SAG1 linked with intrinsically unstructured domain of gra2 protein. Korean J Parasitol 2013; 51: 503-510.

15. Kulldorff M. A spatial scan statistic. Communications in Statistics-Theory and Methods. 1997; 26:1481-1496.

16. Kulldorff M. SaTScan TM: User Guide for version 9.6 [Internet]. Available from: https://www.satscan.org/cgi-bin/satscan/register. pl/SaTScan_Users_Guide.pdf?todo=process_userguide_download.

17. Han JH, Lee MJ. Cancer cluster detection using scan statistic. Journal of the Korean Data \& Information Science Society 2016; 27:1193-1201.

18. Kulldorff M. An isotonic spatial scan statistic for geographical disease surveillance. Journal of the National Institute of Public Health 1999; 48: 94-101.

19. Azage M, Kumie A, Worku A, Bagtzoglou AC. Childhood diarrhea exhibits spatiotemporal variation in northwest Ethiopia: a SaTScan spatial statistical analysis. PLoS One 2015;10: e0144690. 
